\title{
Real-world applicability and impact of early rhythm control for European patients with atrial fibrillation: a report from the ESC-EHRA EORP-AF Long-Term General Registry
}

\author{
Marco Proietti ${ }^{1,2,3}$ - Marco Vitolo ${ }^{1,4}$. Stephanie L. Harrison ${ }^{1}$. Deirdre A. Lane ${ }^{1,5}$. Laurent Fauchier ${ }^{6}$. \\ Francisco Marin ${ }^{7}$. Michael Nabauer ${ }^{8} \cdot$ Tatjana S. Potpara $^{9,10} \cdot$ Gheorghe-Andrei Dan $^{11}$. Giuseppe Boriani ${ }^{4}$ \\ Gregory Y. H. Lip ${ }^{1,5}$ on behalf of the ESC-EHRA EORP-AF Long-Term General Registry Investigators
}

Received: 13 April 2021 / Accepted: 20 July 2021 / Published online: 27 August 2021

(c) The Author(s) 2021

\begin{abstract}
Background Use of rate/rhythm control is essential to control symptoms in patients with atrial fibrillation (AF). Recently, the EAST-AFNET 4 trial described how early rhythm control strategy was associated with a lower risk of adverse clinical outcomes.

Objectives The aim was to evaluate the real-world applicability and impact of an early rhythm control strategy in patients with AF.

Methods Use of an early rhythm control strategy was assessed in a European cohort of AF patients derived from the EHRAESC EORP-AF General Long-Term Registry. Early rhythm control was defined as use of antiarrhythmic drugs or cardioversion/catheter ablation. The primary outcome included cardiovascular death, stroke, acute coronary syndrome, and worsening of heart failure. Quality of life and health-care resource usage were also assessed as outcomes.

Results Among the 10,707 patients evaluated for eligibility to EAST-AFNET 4, a total of 3774 (34.0\%) were included. Early rhythm control was associated with better quality of life, but with greater use of health-care resources. During follow-up, the primary outcome occurred less often in early rhythm control patients than in those with no rhythm control (13.6\% vs. $18.5 \%, p<0.001)$. In the multivariate adjusted Cox regression model, no significant difference was found between no rhythm control and early rhythm control, for the primary outcome. No difference in the primary outcome between early rhythm control and 'no rhythm control patients' adherent to Atrial fibrillation Better Care (ABC) pathway' was evident $(p=0.753)$
\end{abstract}

Giuseppe Boriani, Gregory Y. H. Lip: joint senior authors.

The members of the ESC-EHRA EORP-AF Long-Term General Registry Investigators are listed in "Acknowledgements".

Marco Proietti

marco.proietti@unimi.it

$\triangle$ Gregory Y. H. Lip

gregory.lip@liverpool.ac.uk

1 Liverpool Centre for Cardiovascular Science, University of Liverpool and Liverpool Heart and Chest Hospital, Liverpool, UK

2 Department of Clinical Sciences and Community Health, University of Milan, Milan, Italy

3 Geriatric Unit, IRCCS Istituti Clinici Scientifici Maugeri, Milan, Italy

4 Cardiology Division, Department of Biomedical, Metabolic and Neural Sciences, Policlinico di Modena, University of Modena and Reggio Emilia, Modena, Italy
5 Department of Clinical Medicine, Aalborg University, Aalborg, Denmark

6 Service de Cardiologie, Centre Hospitalier Universitaire Trousseau, Tours, France

7 Department of Cardiology, Hospital Universitario Virgen de la Arrixaca, IMIB-Arrixaca, University of Murcia, CIBERCV, Murcia, Spain

8 Department of Cardiology, Ludwig-Maximilians-University, Munich, Germany

9 School of Medicine, University of Belgrade, Belgrade, Serbia

10 Intensive Arrhythmia Care, Cardiology Clinic, Clinical Center of Serbia, Belgrade, Serbia

11 Colentina University Hospital, 'Carol Davila' University of Medicine, Bucharest, Romania 
Conclusions Use of an early rhythm control strategy was associated with a lower rate of major adverse events, but this difference was non-significant on multivariate analysis, being mediated by differences in baseline characteristics and clinical risk profile. Early rhythm control was associated with a higher use of health-care resources and risk of hospital admission, despite showing better quality of life.

\section{Graphic abstract}

\section{One Third of European AF Patients are} Eligible for Early Rhythm Control

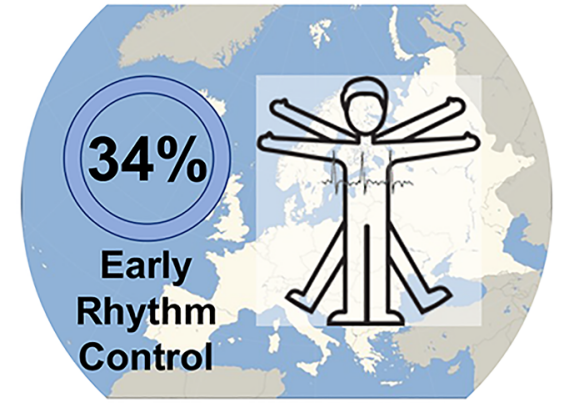

Early Rhythm Control is associated with
more Health-Care Resources Use ( 2-fold)
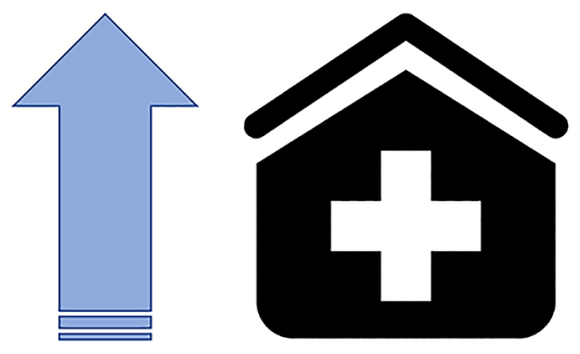

\section{Early Rhythm Control is associated with Higher Risk for Hospitalisation}

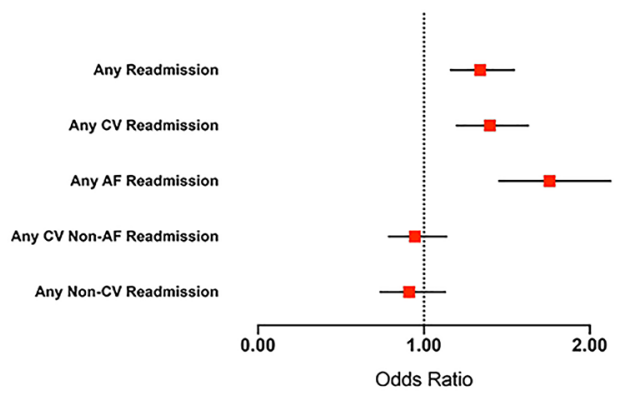

\section{No Advantage for Early Rhythm Control on the Primary Outcome}

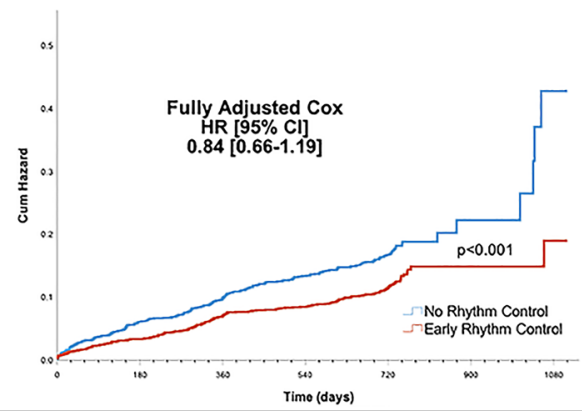

Keywords Atrial fibrillation $\cdot$ Rhythm control $\cdot$ Rate control $\cdot$ Outcomes

\section{Introduction}

Patient-centred, symptom-directed decisions on rate or rhythm control are pivotal considerations for the clinical management of patients with atrial fibrillation (AF). This approach is recommended by the European Society of Cardiology (ESC) 2020 Clinical Guidelines for AF management [1] and is part of the ' $\mathrm{B}$ ' criterion of the Atrial fibrillation Better Care $(\mathrm{ABC})$ pathway for the integrated care management of patients with $\mathrm{AF}$ [2].

It has long been debated whether rate vs. rhythm control strategy could have differential impact on major clinical outcomes, especially the risk of all-cause death occurrence [3-5]. A more recent approach advocated is that a background rate control therapy is needed in all AF patients, with additional rhythm control strategies to mitigate symptom burden $[1,6]$.
The "Early Treatment of Atrial Fibrillation for Stroke Prevention Trial" (EAST-AFNET 4) study [7] tested the hypothesis that early initiation of rhythm control therapy conferred an advantage in terms of the risk of major adverse events. Over a median 5.1 years of follow-up, the trial reported a $21 \%$ relative risk reduction $(6-34 \% \mathrm{CI})$ for a composite primary outcome of cardiovascular (CV) death, stroke, acute coronary syndrome and hospitalization/worsening of heart failure [7]. Given that the intervention arm had structured follow-up and a 'package of care', the applicability of this trial to real world clinical practice requires further study.

In this ancillary analysis from the ESC-European Heart Rhythm Association (EHRA) EURObservational Research Programme (EORP) AF General Long-Term Registry, the aim of this study was to utilize a large real-world cohort of European AF patients: first, to determine how many patients would be able to fulfil the 
EAST-AFNET 4 inclusion criteria; and second, to test the impact of an early rhythm control strategy on the use of health care resources, quality of life and major adverse events.

\section{Methods}

The ESC-EHRA EORP AF General Long-Term Registry is a multicentre observational registry held by the ESC and endorsed by the EHRA, with the General Long-Term Registry preceded by the EORP-AF General Pilot Registry [8-11]. The EORP-AF General Long-Term Registry is a prospective, observational, multicentre registry established by ESC in 27 participating countries. The study enrolled consecutive patients with AF presenting in 250 cardiology practices, in both in- and outpatient settings. The detailed description of the study design, baseline characteristics and 1-year follow-up results have been provided previously $[12,13]$. Briefly, all patients enrolled had AF documented within 12 months before enrolment on the basis of objective electrocardiographic evaluation. All patients were aged $\geq 18$ years and provided written informed consent. Enrolment was undertaken from October 2013 to September 2016, with 1-year and 2-year follow-up. Institutional review board approved the study protocol for each country, and the study was performed according to the EU Note for Guidance on Good Clinical Practice CPMP/ECH/135/95 and the Declaration of Helsinki.

Symptomatic status was defined according to EHRA score [1]. Thromboembolic risk was defined according to the $\mathrm{CHA}_{2} \mathrm{DS}_{2}$-VASc score [14], with bleeding risk defined by the HAS-BLED score [15]. Multimorbidity was defined as the concomitant presence of at least two different long-term comorbidities. Frailty was defined on the basis of a 38-items frailty index of $\geq 0.25$, according to Rockwood and Mitnitski [16]. Polypharmacy was classified as the concomitant prescription of $\geq 5$ drugs [17]. Adherence of clinical management to the 'Atrial fibrillation Better Care' (ABC) pathway was retrospectively defined in this cohort, as previously reported [18]. Briefly, the ABC pathway has been proposed to streamline integrated care and holistic management in $\mathrm{AF}$ patients and is based on the following: (i) avoid stroke with anticoagulation; (ii) better symptom management with patient-centred symptom-directed decisions on rate or rhythm control; (iii) cardiovascular risk factor and comorbidities optimization including lifestyle changes [2]. Adherence to the ABC pathway has consistently been associated with reduction in risk for major clinical outcomes associated with AF [19].

\section{Evaluation of eligibility to EAST-AFNET 4 trial criteria}

To understand how many patients in the current cohort would have been eligible to be enrolled in the EAST-AFNET 4 , we applied the same inclusion criteria to our population [20]. All the patients included in the EORP-AF General Long-Term Registry were $\geq 18$ years and diagnosed with AF within 12 months of enrolment. Hence, we included all the patients that were aged $\geq 75$ years or had a clinical history positive for previous stroke or transient ischaemic attack. Additionally, we included all the patients that fulfilled at least two of the following criteria: (i) age $\geq 65$ years; (ii) female sex; (iii) hypertension; (iv) diabetes mellitus; (v) previous myocardial infarction or any coronary revascularization procedure; (vi) stable heart failure (NYHA II or ejection fraction $<50 \%$ ); (vii) left ventricular hypertrophy: (viii) chronic kidney disease (creatinine clearance 15-59 mL/ $\min$ ); (ix) peripheral arterial disease.

\section{Definition of early rhythm control}

Early rhythm control was defined at the moment of enrolment visit or discharge following hospital admission. All those patients who received a rhythm control intervention during the index episode, such as electrical cardioversion, pharmacological cardioversion, catheter ablation, or were prescribed an antiarrhythmic drug (Class Ia, Class Ic, Class III) at discharge, were included in the 'early rhythm control' group.

All the other patients prescribed beta blockers, digoxin, or non-dihydropyridine calcium-channel blockers, as rate control drugs, were included in the 'no rhythm control' group.

\section{Evaluation of quality of life}

Quality of life was evaluated at baseline and 1-year followup using the EQ-5D-5L questionnaire, a generic, extensively validated, easy to use instrument that consists of two parts: the EQ-5D descriptive system and the EQ visual analogue scale (https://euroqol.org/eq-5d-instruments/eq-5d-51about/). The descriptive system consists of five dimensions (mobility, self-care, usual activities, pain/discomfort and anxiety/depression) with five possible levels for each dimension (no problems, slight problems, moderate problems, severe problems and extreme problems), generating $5^{5}=3125$ unique health states. According to a previous report, using the UK trade-off value set we translated each of the levels into a single numeric value, with the lowest values corresponding to better health [21]. Furthermore, combining the single values, we translated the five-digit health state into a single index, the Health Utility Score (HUS) by subtracting each value from 1 . The best possible health in 
each dimension $(=11,111)$ corresponded to an HUS of 1.0 (perfect health). An HUS of 0 is equivalent to death. The visual analogue scale was used for patients to self-rate their current health status, ranging from 0 (worst health imaginable) to 100 (best health imaginable).

\section{Evaluation of health-care resources use}

We examined the differential use of health-care resources according to the use of early rhythm control. In patients enrolled during hospitalization, we evaluated the overall length of stay. Further, we analysed the occurrence and number of cardiology and internal medicine/general practitioner visits, as well as the emergency room (ER) admissions during the follow-up observation (at 1 and 2 years of follow-up).

Also, to account comprehensively for the use of healthcare resources, we evaluated the occurrence of hospital admission/readmission throughout follow-up observation, as follows: (i) any readmission; (ii) any $\mathrm{CV}$ readmission; (iii) any AF readmission; (iv) any $\mathrm{CV}$ non-AF readmission; and (v) any non-CV readmission.

\section{Major adverse events}

The primary main outcome of the study was equivalent to the original EAST-AFNET 4 trial primary outcome, being a composite of (i) CV death; (ii) any stroke; (iii) worsening heart failure; and (iv) acute coronary syndrome. The secondary clinical outcomes were: (i) major adverse cardiovascular events (MACEs): as the composite of any thromboembolic events, any acute coronary syndrome and CV death; and (ii) all-cause death.

\section{Statistical analysis}

Continuous variables were expressed as mean SD or median IQR and differences across the groups were evaluated according to Student's $T$ test and Mann-Whitney $U$ test, respectively. Categorical variables were expressed as counts and percentages and differences across groups were evaluated according to the Chi-square test. A univariate and multivariate logistic regression model was compiled to evaluate the clinical factors associated with the choice of early rhythm control.

To evaluate the relationship between the use of early rhythm control and length of hospital stay, in addition to the number of medical visits during the follow-up, we performed a linear regression analysis adjusted for type of AF, $\mathrm{CHA}_{2} \mathrm{DS}_{2}$-VASc score and EHRA score. To analyse the association between the use of early rhythm control and the occurrence of medical visits, a logistic regression model was used, adjusted for type of $\mathrm{AF}, \mathrm{CHA}_{2} \mathrm{DS}_{2}$-VASc score and EHRA score. We also performed a linear regression analysis adjusted for type of AF, $\mathrm{CHA}_{2} \mathrm{DS}_{2}$-VASc score and EHRA score to evaluate the relationship between the use of early rhythm control and quality of life measures at baseline and throughout follow-up. The association between the use of early rhythm control and readmission outcomes was tested using a logistic regression model, adjusted for type of AF, $\mathrm{CHA}_{2} \mathrm{DS}_{2}$-VASc score, EHRA score and use of OAC.

Differences in cumulative risk for the main study outcome were evaluated using log-rank tests and Kaplan-Meier curves. To investigate the independent associations between the use of early rhythm control and the primary and main secondary clinical outcomes, a Cox regression analysis was performed. Two different models were constructed: (i) adjusted for type of $\mathrm{AF}, \mathrm{CHA}_{2} \mathrm{DS}_{2}$-VASc score and EHRA score and use of oral anticoagulant (OAC); (ii) adjusted for type of AF, EHRA score, age, sex, hypertension, diabetes mellitus, heart failure, severe coronary artery disease, valvular disease, left ventricular hypertrophy, peripheral artery disease, stroke/transient ischaemic attack, chronic kidney disease, chronic obstructive pulmonary disease, malignancy and use of OAC. Lastly, we performed a sensitivity analysis regarding the occurrence of study primary outcome, comparing the early rhythm control strategy to a "no rhythm control' approach adherent to the $\mathrm{ABC}$ pathway.

All linear regression analyses were reported as Beta coefficient and 95\% CI. All logistic regression analyses results were reported as OR and 95\% CI. All Cox regression analyses results were reported as HR and 95\% CI. A two-sided $p<0.05$ was considered statistically significant. All analyses were performed using SPSS statistical software version 25.0.0.1 (IBM, NY, USA) for MacOS.

\section{Results}

From the original 11,096 patients enrolled in the EORPAF, 10,707 (96.5\%) were evaluated for eligibility to EASTAFNET 4 inclusion criteria, due to missing data for the variables use to evaluate eligibility. Overall, 3774 (34.0\%) patients fulfilled the study's inclusion criteria and were selected for this analysis. Among those not selected, 2654 (38.3\%) did not fulfil the study main criteria; 3682 (53.1\%) did not fulfil the study additional criteria; 4013 (57.9\%) had long-standing persistent or permanent AF or did not report the type of AF.

Comparing EORP-AF patients included in this analysis to those not included, included patients were older, with higher $\mathrm{CHA}_{2} \mathrm{DS}_{2}$-VASc and HAS-BLED scores and with more comorbidities and prescribed medications (all $p<0.001$ ). EORP-AF patients qualifying for inclusion had a higher proportion of those aged $\geq 75$ years, were more likely to have persistent AF and had a higher symptom burden compared to those enrolled in the EAST-AFNET 4 study 
(Table 1). Also, EAST-AFNET 4 patients reported higher proportions of hypertension and diabetes mellitus, while EORP-AF patients had more structural heart disease (heart failure, severe coronary artery disease, valvular disease, left ventricular hypertrophy) (Table 1).

\section{Determinants of early rhythm control}

Among EORP-AF patients, 2052 (54.4\%) were treated according to an early rhythm control strategy and 1722 (45.6\%) with no rhythm control at baseline. Patients prescribed early rhythm control were younger, more likely male, with persistent AF and a higher burden of AF symptoms. The early rhythm control group had more prevalent hypertension, but less comorbidities, with lower thromboembolic and bleeding risk factors, and a greater proportion receiving OAC (Table 1). Mean (SD) time from AF diagnosis to enrolment was not significantly different between patients treated with and without early rhythm control [48.6 (84.4) days vs. 51.2 (88.6) days, respectively; $p=0.426]$, nor was the mean (SD) time from enrolment to discharge [0.4 (14.1) days vs. 1.5 (16.7) days, respectively; $p=0.381]$.

At discharge, among patients not prescribed early rhythm control, a rate control strategy based on the use of beta blockers was most commonly used (63.3\%), while $29.0 \%$ were managed with a combination of rate control drugs and only a minority were treated with digoxin only $(2.1 \%)$ and non-dihydropyridine calcium-channel blockers $(5.5 \%)$. (Fig. 1). In the early rhythm control group, use of a combination antiarrhythmic strategy (antiarrhythmic drugs + cardioversion/ablation) was most common (36.1\%), with sole use of antiarrhythmic drugs only in $28.2 \%$ (Fig. 1). Electric cardioversion only was used in $22.5 \%$ of patients, pharmacological cardioversion only in $9.9 \%$ and catheter ablation only in $3.3 \%$ (Fig. 1).

Based on the differences in baseline characteristics, univariate logistic regression analysis for the clinical factors associated with the use of an early rhythm control strategy, followed by a multivariate model (Table S1), showed that paroxysmal and persistent $\mathrm{AF}$, progressively increasing symptom burden and presence of hypertension were significantly associated with the use of an early rhythm control strategy. Increasing age, higher NYHA class and concomitant diabetes mellitus, stroke/transient ischaemic attack and chronic obstructive pulmonary disease were all inversely associated with early rhythm control (Table S1).

\section{Evaluation of quality of life}

At baseline, evaluation of quality of life showed that patients managed with early rhythm control were more able to attend to their self-care $(p=0.001)$ and their usual activities $(p<0.001)$, but were more likely anxious, even after adjustment for $\mathrm{CHA}_{2} \mathrm{DS}_{2}$-VASc score, type of $\mathrm{AF}$ and EHRA score (Table 2). In the multivariate analysis, at 1-year follow-up, those patients with early rhythm control had better levels of mobility $(p<0.001)$, self-care $(p=0.009)$ and participation in their usual activities $(p=0.005)$, with an overall better health state according to the HUS $(p=0.002)$, compared to those not prescribed with an early rhythm control. At 2 years follow-up, patients prescribed early rhythm control reported less impairment in all five dimensions of the EQ-5D-5L questionnaire and a better overall health state according to both HUS $(p<0.001)$ and VAS $(p=0.053)$, after adjustment for $\mathrm{CHA}_{2} \mathrm{DS}_{2}$-VASc score, type of $\mathrm{AF}$ and EHRA score (Table 2).

\section{Evaluation of health-care resources use}

Use of health-care resources is reported in Table 3. Patients managed with early rhythm control attended more cardiology and internal medicine/general practitioner appointments at both follow-up time points (all $p<0.001$ ) (Table 3, upper panel).

Among hospitalized patients, those prescribed early rhythm control had a shorter mean hospital stay, even after adjustment ( $p=0.001)$ (Table 3, lower panel). Among those who attended cardiology visits, those with early rhythm control reported a higher number of visits both at 1 year $(p<0.001)$ and 2 years $(p=0.023)$ of follow-up. Among patients attending internal medicine/general practitioner visits, those with early rhythm control attended more often within the first year of follow-up than those with "no rhythm control' $(p=0.015)$.

Patients treated with an early rhythm control strategy had a higher rate of any readmission, and admissions related to $\mathrm{CV}$ reasons or AF (all $p<0.001$ ) (Table 3). Logistic regression, adjusted for type of AF, EHRA score, $\mathrm{CHA}_{2} \mathrm{DS}_{2}-\mathrm{VASc}$ score and use of OAC, found that an early rhythm control strategy was associated with higher odds of any hospital readmission, any $\mathrm{CV}$ readmission and any $\mathrm{AF}$ readmission (Table 3).

\section{Major adverse events}

Follow-up data were available for 3354 (88.9\%) of the patients. Over a mean (SD) follow-up of 675.4 (181.3) days, a total of 532 (14.1\%) EAST-AFNET 4 defined primary outcome events were reported with an overall incidence of 8.9 per 100 patient-years. Death occurred in 321 $(8.5 \%)$ and $380(10.1 \%)$ experienced MACEs. Compared to those treated with an early rhythm control approach, the no rhythm control group had a higher proportion of patients with the EAST-AFNET 4 defined primary outcome, MACEs and all-cause death events (Table 4). The incidence of EAST-AFNET 4 defined primary outcome 
Table 1 Baseline characteristics in EAST-AFNET 4 and EORP-AF and according to early rhythm control prescription

\begin{tabular}{|c|c|c|c|c|c|}
\hline Variables & EAST-AFNET $4(N=2789)$ & EORP-AF $(N=3774)$ & $\begin{array}{l}\text { No rhythm control } \\
(N=1722)\end{array}$ & $\begin{array}{l}\text { Early rhythm con- } \\
\text { trol }(N=2052)\end{array}$ & $p^{*}$ \\
\hline Age, years mean (SD) & $70.3(8.3)$ & $69.8(10.4)$ & $71.9(10.3)$ & $68.0(10.2)$ & $<0.001$ \\
\hline Age, years median [IQR] & $71[66-76]$ & $71[63-78]$ & $74[66-79]$ & $69[62-76]$ & $<0.001$ \\
\hline Age $\geq 75$ years, $n(\%)$ & $812(29.1)$ & $1483(39.3)$ & 855 (49.7) & $628(30.6)$ & $<0.001$ \\
\hline Male sex, $n(\%)$ & $1496(53.6)$ & $2026(53.7)$ & $878(51.0)$ & $1148(55.9)$ & 0.002 \\
\hline BMI, $\mathrm{kg} / \mathrm{m}^{2}$ median [IQR] & $28.6[25.5-32.1]$ & $27.8[24.8-31.2]$ & $27.5[24.6-31.2]$ & $27.9[25.0-31.3]$ & 0.024 \\
\hline Type of AF, $n(\%)$ & & & & & $<0.001$ \\
\hline First detected & $1048(37.6)$ & $1031(27.3)$ & $564(32.8)$ & $467(22.8)$ & \\
\hline Paroxysmal & 994 (35.7) & $1538(40.8)$ & $742(43.1)$ & $796(38.8)$ & \\
\hline Persistent & 743 (26.7) & $1205(31.9)$ & $416(24.2)$ & $789(38.5)$ & \\
\hline EHRA score, $n(\%)$ & & & & & $<0.001$ \\
\hline EHRA I & $801(30.4)$ & $1390(36.8)$ & $784(45.5)$ & $606(29.5)$ & \\
\hline EHRA II & $1358(51.6)$ & $1465(38.8)$ & $581(33.7)$ & $884(43.1)$ & \\
\hline EHRA III & $447(17.0)$ & $808(21.4)$ & $310(18.0)$ & $498(24.3)$ & \\
\hline EHRA IV & $27(1.0)$ & $110(2.9)$ & $47(2.7)$ & $63(3.1)$ & \\
\hline Hypertension, $n(\%)$ & $2450(87.8)$ & $2559(68.0)$ & $1122(65.4)$ & $1437(70.1)$ & 0.002 \\
\hline Diabetes mellitus, $n(\%)$ & $694(24.9)$ & $860(22.8)$ & $440(25.6)$ & $420(20.5)$ & $<0.001$ \\
\hline NYHA class, $n(\%)$ & & & & & $<0.001$ \\
\hline No HF & $1819(65.3)$ & $2360(62.5)$ & $1019(59.2)$ & $1341(65.4)$ & \\
\hline I & 331 (11.9) & $239(6.3)$ & $125(7.3)$ & $114(5.6)$ & \\
\hline II & $514(18.5)$ & $712(18.9)$ & $325(18.9)$ & $387(18.9)$ & \\
\hline III & $120(4.3)$ & $396(10.5)$ & $214(12.4)$ & $182(8.9)$ & \\
\hline IV & - & $67(1.8)$ & $39(2.3)$ & $28(1.4)$ & \\
\hline Prior stroke/TIA, $n(\%)$ & $328(11.8)$ & $379(10.1)$ & $191(11.2)$ & $188(9.2)$ & 0.049 \\
\hline Severe CAD, $n(\%)$ & 479 (17.2) & 827 (21.9) & $392(22.8)$ & $435(21.2)$ & 0.247 \\
\hline Valvular disease, $n(\%)$ & $1251(45.0)$ & 1807 (48.6) & $847(50.3)$ & $960(47.2)$ & 0.064 \\
\hline $\mathrm{LVH}, n(\%)$ & $132(4.7)$ & $988(28.5)$ & $463(30.1)$ & $525(27.3)$ & 0.070 \\
\hline $\mathrm{PAD}, n(\%)$ & $122(4.4)$ & $303(8.1)$ & $142(8.4)$ & $161(7.9)$ & 0.608 \\
\hline $\mathrm{CKD}, n(\%)$ & $351(12.6)$ & $454(12.0)$ & 234 (13.6) & $220(10.7)$ & 0.006 \\
\hline COPD, $n(\%)$ & $209(7.5)$ & $279(7.4)$ & $168(9.8)$ & $111(5.4)$ & $<0.001$ \\
\hline Malignancy, $n(\%)$ & & & & & 0.003 \\
\hline No malignancy & $2563(92.2)$ & 3467 (91.9) & $1560(90.6)$ & $1907(92.9)$ & \\
\hline Active malignancy & $19(0.7)$ & $79(2.1)$ & $52(3.0)$ & $27(1.3)$ & \\
\hline Prior malignancy & $197(7.1)$ & $216(5.7)$ & $104(6.0)$ & $112(5.5)$ & \\
\hline $\mathrm{CHA}_{2} \mathrm{DS}_{2}$-VASc, mean (SD) & $3.3(1.3)$ & $3.3(1.6)$ & $3.6(1.6)$ & $3.1(1.5)$ & $<0.001$ \\
\hline $\mathrm{CHA}_{2} \mathrm{DS}_{2}$-VASc, median [IQR] & $3[2-4]$ & $3[2-4]$ & $4[2-5]$ & $3[2-4]$ & $<0.001$ \\
\hline HAS-BLED, mean (SD) & - & $1.6(1.0)$ & $1.7(1.1)$ & $1.5(1.0)$ & $<0.001$ \\
\hline HAS-BLED, median [IQR] & - & $2[1,2]$ & $2[1,2]$ & $1[1,2]$ & $<0.001$ \\
\hline Multimorbidity, $n(\%)$ & - & $2681(83.3)$ & $1218(84.3)$ & $1463(82.4)$ & 0.158 \\
\hline Frailty, $n(\%)$ & - & $555(21.7)$ & $236(20.6)$ & $319(22.5)$ & 0.246 \\
\hline Polypharmacy, $n(\%)$ & - & $2375(63.3)$ & $999(58.3)$ & $1376(67.5)$ & $<0.001$ \\
\hline ABC pathway adherence, $n(\%)$ & - & $758(29.9)$ & $344(29.3)$ & $414(30.5)$ & 0.483 \\
\hline ABC pathway criteria, $n(\%)$ & - & & & & 0.335 \\
\hline 0 & & $59(2.3)$ & $33(2.8)$ & $26(1.9)$ & \\
\hline 1 & & $494(19.5)$ & $239(20.3)$ & $255(18.8)$ & \\
\hline 2 & & $1221(48.2)$ & $560(47.6)$ & $661(48.7)$ & \\
\hline 3 & & $758(29.9)$ & $344(29.3)$ & $414(30.5)$ & \\
\hline \multicolumn{6}{|l|}{ Pharmacological treatments } \\
\hline Any antiplatelet, $n(\%)$ & $455(16.4)$ & $921(24.4)$ & $441(25.6)$ & $480(23.4)$ & 0.111 \\
\hline Any OAC, $n(\%)$ & $2517(90.5)$ & $3255(86.3)$ & $1426(82.8)$ & $1829(89.2)$ & $<0.001$ \\
\hline
\end{tabular}


Table 1 (continued)

\begin{tabular}{|c|c|c|c|c|c|}
\hline Variables & EAST-AFNET $4(N=2789)$ & EORP-AF $(N=3774)$ & $\begin{array}{l}\text { No rhythm control } \\
(N=1722)\end{array}$ & $\begin{array}{l}\text { Early rhythm con- } \\
\text { trol }(N=2052)\end{array}$ & $p^{*}$ \\
\hline Any VKA, $n(\%)$ & - & $1644(43.6)$ & $687(39.9)$ & $957(46.7)$ & $<0.001$ \\
\hline Any NOAC, $n(\%)$ & - & $1613(42.8)$ & $740(43.0)$ & 873 (42.6) & 0.777 \\
\hline ACEi/ARBs, $n(\%)$ & $1932(69.4)$ & $2541(67.4)$ & $1156(67.3)$ & $1385(67.5)$ & 0.875 \\
\hline Diuretics, $n(\%)$ & $1120(40.3)$ & $1900(50.4)$ & $902(52.5)$ & 998 (48.7) & 0.020 \\
\hline MRAs, $n(\%)$ & $182(6.5)$ & $577(15.3)$ & $276(16.1)$ & $301(14.7)$ & 0.240 \\
\hline Statins, $n(\%)$ & $1196(43.0)$ & $1832(48.6)$ & $843(49.0)$ & $989(48.3)$ & 0.636 \\
\hline Oral antidiabetics, $n(\%)$ & $459(16.5)$ & $599(15.9)$ & $314(18.3)$ & $285(13.9)$ & $<0.001$ \\
\hline Insulin, $n(\%)$ & $121(4.3)$ & $205(5.4)$ & $102(5.9)$ & $103(5.0)$ & 0.220 \\
\hline
\end{tabular}

$A B C$ atrial fibrillation better care, $A F$ atrial fibrillation, $B M I$ body mass index, $C A D$ coronary artery disease, $C K D$ chronic kidney disease, $C O P D$ chronic obstructive pulmonary disease, EHRA European Heart Rhythm Association, $H F$ heart failure, IQR interquartile range, $L V H$ left ventricular hypertrophy, NYHA New York Heart Association, PAD peripheral artery disease, SD standard deviation, TIA transient ischaemic attack

* $p$ value is referred to the comparison between no rhythm control and early rhythm control

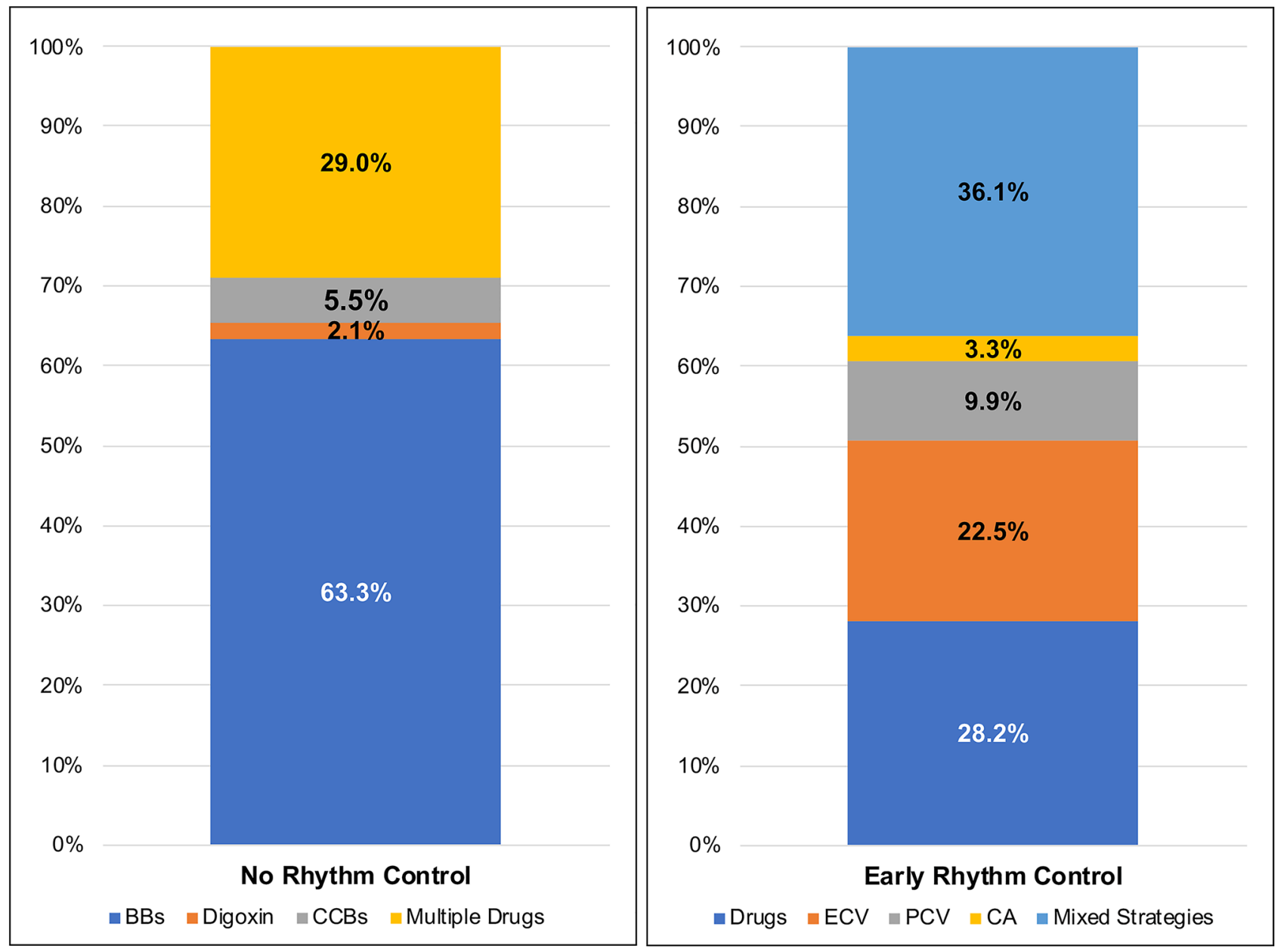

Fig. 1 Distribution of rate/rhythm control treatments according to groups at baseline. $B B s$ beta blockers, $C A$ catheter ablation, $C C B s$ calciumchannel blockers, $E C V$ electric cardioversion, $P C V$ pharmacological cardioversion 
Table 2 Quality of life indicators according to early rhythm control

$\begin{array}{llll}\text { No rhythm control } & \text { Early rhythm control } & p & \begin{array}{l}\text { Early rhythm control } \\ \text { Beta }(95 \% \mathrm{CI})^{*}\end{array}\end{array}$

Baseline

\begin{tabular}{|c|c|c|c|c|c|}
\hline EQ-5D-5L mobility & $0.049(0.063)$ & $0.043(0.061)$ & 0.019 & $-0.003(-0.007 / 0.001)$ & 0.156 \\
\hline EQ-5D-5L self-care & $0.020(0.039)$ & $0.014(0.035)$ & $<0.001$ & $-0.005(0.007 /-0.002)$ & 0.001 \\
\hline EQ-5D-5L usual activities & $0.037(0.047)$ & $0.031(0.044)$ & $<0.001$ & $-0.006(-0.009 /-0.003)$ & $<0.001$ \\
\hline EQ-5D-5L pain/discomfort & $0.049(0.068)$ & $0.044(0.063)$ & 0.024 & $-0.004(-0.009 / 0.000)$ & 0.062 \\
\hline EQ-5D-5L anxiety & $0.046(0.066)$ & $0.054(0.072)$ & 0.001 & $0.007(0.002 / 0.012)$ & 0.004 \\
\hline Health utility score & $0.80(0.21)$ & $0.81(0.19)$ & 0.068 & $0.010(-0.004 / 0.025)$ & 0.142 \\
\hline Visual analogue scale & $68.3(20.6)$ & $68.3(20.8)$ & 0.992 & $0.093(-1.486 / 1.673)$ & 0.908 \\
\hline \multicolumn{6}{|l|}{-year follow-up } \\
\hline EQ-5D-5L mobility & $0.046(0.059)$ & $0.036(0.056)$ & $<0.001$ & $-0.009(-0.014 /-0.004)$ & $<0.001$ \\
\hline EQ-5D-5L self-care & $0.018(0.035)$ & $0.013(0.033)$ & 0.002 & $-0.004(-0.007 /-0.001)$ & 0.009 \\
\hline EQ-5D-5L usual activities & $0.033(0.042)$ & $0.027(0.041)$ & 0.001 & $-0.005(-0.009 /-0.002)$ & 0.005 \\
\hline EQ-5D-5L pain/discomfort & $0.044(0.067)$ & $0.039(0.062)$ & 0.056 & $-0.005(-0.010 / 0.001)$ & 0.118 \\
\hline EQ-5D-5L anxiety & $0.042(0.066)$ & $0.040(0.057)$ & 0.536 & $-0.004(-0.009 / 0.002)$ & 0.197 \\
\hline Health utility score & $0.817(0.203)$ & $0.844(0.185)$ & 0.001 & $0.026(0.009 / 0.043)$ & 0.002 \\
\hline Visual analog scale & $69.7(19.8)$ & $70.9(19.8)$ & 0.169 & $1.283(-0.505 / 3.071)$ & 0.159 \\
\hline \multicolumn{6}{|l|}{ years follow-up } \\
\hline EQ-5D-5L mobility & $0.051(0.062)$ & $0.040(0.056)$ & $<0.001$ & $-0.009(-0.015 /-0.004)$ & 0.001 \\
\hline EQ-5D-5L self-care & $0.021(0.040)$ & $0.012(0.027)$ & $<0.001$ & $-0.009(-0.012 /-0.005)$ & $<0.001$ \\
\hline EQ-5D-5L usual activities & $0.038(0.045)$ & $0.028(0.041)$ & $<0.001$ & $-0.008(-0.012 /-0.004)$ & $<0.001$ \\
\hline EQ-5D-5L pain/discomfort & $0.048(0.068)$ & $0.039(0.062)$ & 0.004 & $-0.008(-0.014 /-0.001)$ & 0.016 \\
\hline EQ-5D-5L anxiety & $0.043(0.065)$ & $0.037(0.058)$ & 0.052 & $-0.007(-0.013 /-0.001)$ & $\mathbf{0 . 0 2 0}$ \\
\hline Health utility score & $0.80(0.21)$ & $0.84(0.18)$ & $<0.001$ & $0.042(0.023 / 0.060)$ & $<0.001$ \\
\hline Visual analogue scale & $69.8(19.4)$ & $72.1(19.8)$ & 0.016 & $1.943(-0.026 / 3.911)$ & 0.053 \\
\hline
\end{tabular}

Bold values depict significant association

CI confidence interval; for other acronyms please see previous tables' legends

*Adjusted for $\mathrm{CHA}_{2} \mathrm{DS}_{2}$-VASc score, type of AF, EHRA score

was 10.8 per 100 patient-years in patients with no rhythm control and 7.4 per 100 patient-years in patients with early rhythm control. Kaplan-Meier curves for the EASTAFNET 4 trial defined primary outcome showed that an early rhythm control strategy was associated with a progressively lower cumulative risk $(p<0.001)$ (Fig. 2).

Finally, a Cox regression analysis was performed to establish the association between use of an early rhythm control strategy and the risks of primary and secondary outcomes (Table 5). While the univariate analysis showed that prescription of an early rhythm control strategy was associated with a lower risk for all primary and secondary outcomes, the progressive adjustment process (Model 1 adjusted for type of AF, EHRA score, $\mathrm{CHA}_{2} \mathrm{DS} 2$-VASc score and use of OAC; Model 2 adjusted for type of AF EHRA score, all comorbidities evaluated at baseline and use of OAC) showed a gradual and subsequent loss in association, with the fully adjusted Model 2 showing no significant differences between patients treated with and without an early rhythm control strategy (HR $0.84,95 \%$ CI
0.66-1.19 for EAST-AFNET 4 defined primary outcome; HR $0.95,95 \%$ CI $0.73-1.12$ for MACEs; HR $0.96,95 \%$ CI $0.75-1.24$ for all-cause death).

In the final multivariable model for the EAST-AFNET 4 defined primary outcome, heart failure was associated with a nonsignificant trend for increased risk (HR: 1.21, 95\% CI $0.98-1.50, p=0.075$ ). For the occurrence of MACEs, paroxysmal AF was associated with an increased risk of events compared to persistent AF (HR: 1.42, 95\% CI 1.02-1.98, $p=0.040)$.

\section{Sensitivity analysis}

We conducted a sensitivity analysis comparing the occurrence of the primary outcome in patients managed with early rhythm control and those with no rhythm control approach but adherent to the $\mathrm{ABC}$ pathway management. We selected all patients with data on ABC pathway adherence (2532, $67.1 \%$ ) and compared those managed with early rhythm control $(1356,53.6 \%)$, to those not treated with rhythm control 
Table 3 Use of health-care resources according to early rhythm control use

\begin{tabular}{|c|c|c|c|c|c|}
\hline & No rhythm control & Early rhythm control & $p$ & $\begin{array}{l}\text { Early rhythm control } \\
\text { OR }(95 \% \mathrm{CI})^{*}\end{array}$ & $p$ \\
\hline Cardiology visits $1 \mathrm{Y}, n(\%)$ & $940(68.3)$ & $1386(83.0)$ & $<0.001$ & $2.07(1.73-2.47)$ & $<0.001$ \\
\hline IM/GP visits $1 \mathrm{Y}, n(\%)$ & $481(43.9)$ & $822(58.9)$ & $<0.001$ & $1.87(1.58-2.21)$ & $<0.001$ \\
\hline ER admissions $1 \mathrm{Y}, n(\%)$ & $290(21.6)$ & $371(22.4)$ & 0.577 & $1.08(0.90-1.29)$ & 0.422 \\
\hline Cardiology visits $2 \mathrm{Y}, n(\%)$ & $738(61.6)$ & $1130(76.2)$ & $<0.001$ & $1.88(1.58-2.23)$ & $<0.001$ \\
\hline IM/GP visits $2 \mathrm{Y}, n(\%)$ & $430(42.1)$ & $760(58.9)$ & $<0.001$ & $2.02(1.70-2.40)$ & $<0.001$ \\
\hline \multirow[t]{2}{*}{ ER admissions $2 \mathrm{Y}, n(\%)$} & $181(15.8)$ & $242(16.8)$ & 0.497 & $1.08(0.87-1.35)$ & 0.482 \\
\hline & No rhythm control & Early rhythm control & $p$ & $\begin{array}{l}\text { Early rhythm control } \\
\text { Beta }(95 \% \mathrm{CI})^{*}\end{array}$ & $p$ \\
\hline Length of stay, days mean (SD) & $7.6(7.1)$ & $5.9(6.7)$ & $<0.001$ & $-0.992(-1.572 /-0.412)$ & 0.001 \\
\hline Cardiology visits $1 \mathrm{Y}, N$ mean (SD) & $2.4(1.9)$ & $2.7(2.2)$ & $<0.001$ & $0.349(0.168 / 0.530)$ & $<0.001$ \\
\hline IM/GP visits $1 \mathrm{Y}, N$ mean (SD) & $3.9(3.4)$ & $4.6(3.9)$ & 0.004 & $0.589(0.115 / 1.062)$ & 0.015 \\
\hline ER admissions $1 \mathrm{Y}, N$ mean (SD) & $1.7(1.3)$ & $1.7(1.4)$ & 0.704 & $0.005(-0.209 / 0.219)$ & 0.963 \\
\hline Cardiology visits $2 \mathrm{Y}, N$ mean (SD) & $2.0(1.4)$ & $2.2(1.8)$ & 0.004 & $0.184(0.025 / 0.342)$ & $\mathbf{0 . 0 2 3}$ \\
\hline IM/GP visits $2 \mathrm{Y}, N$ mean $(\mathrm{SD})$ & $3.5(3.4)$ & $3.7(3.3)$ & 0.505 & $0.094(-0.348 / 0.536)$ & 0.676 \\
\hline \multirow[t]{2}{*}{ ER admissions $2 \mathrm{Y}, N$ mean (SD) } & $1.6(1.2)$ & $1.6(1.3)$ & 0.610 & $0.061(-0.193 / 0.315)$ & 0.637 \\
\hline & No rhythm control & Early rhythm control & $p$ & $\begin{array}{l}\text { Early rhythm control } \\
\text { OR }(95 \% \text { CI })^{\dagger}\end{array}$ & $p$ \\
\hline Any readmission, $n(\%)$ & $624(38.6)$ & $857(46.2)$ & $<0.001$ & $1.34(1.16-1.54)$ & $<\mathbf{0 . 0 0 1}$ \\
\hline Any $\mathrm{CV}$ readmission, $n(\%)$ & $415(25.7)$ & $607(32.7)$ & $<0.001$ & $1.40(1.20-1.63)$ & $<\mathbf{0 . 0 0 1}$ \\
\hline Any AF readmission, $n(\%)$ & $205(12.7)$ & $408(22.0)$ & $<0.001$ & $1.76(1.45-2.12)$ & $<0.001$ \\
\hline Any $\mathrm{CV}$ non-AF readmission, $n(\%)$ & $303(18.8)$ & $317(17.1)$ & 0.203 & $0.95(0.79-1.14)$ & 0.540 \\
\hline Any non-CV readmission, $n(\%)$ & $207(12.8)$ & $213(11.5)$ & 0.232 & $0.91(0.74-1.13)$ & 0.369 \\
\hline
\end{tabular}

Bold values depict significant association

$1 Y$ 1-year follow-up, $2 Y R 2$ years follow-up, ER emergency room, GP general practitioner, $I M$ internal medicine, $O R$ odds ratio; for other acronyms please see previous tables' legends.

*Adjusted for $\mathrm{CHA}_{2} \mathrm{DS}_{2}$-VASc score, type of AF, EHRA score

${ }^{\dagger}$ Adjusted for $\mathrm{CHA}_{2} \mathrm{DS}_{2}$-VASc score, type of AF, EHRA score, use of OAC

Table 4 Major clinical outcomes comparing early rhythm control versus no rhythm control in the EORP-AF registry patients eligible for EAST-AFNET 4

\begin{tabular}{lllr}
\hline$N(\%)$ & No rhythm control & Early rhythm control & $p$ \\
\hline $\begin{array}{l}\text { EAST-AFNET } \\
\text { 4 primary }\end{array}$ & $287(18.5)$ & $245(13.6)$ & $<0.001$ \\
$\begin{array}{l}\text { outcome } \\
\text { MACEs }\end{array}$ & $204(12.5)$ & $176(9.4)$ & 0.003 \\
All-cause death & $191(11.9)$ & $130(6.7)$ & $<0.001$ \\
\hline
\end{tabular}

$A F$ atrial fibrillation, $C V$ cardiovascular, MACEs major adverse cardiovascular events

but managed with a clinical management adherent to the ABC pathway $(344,13.6 \%)$.

During follow-up, no difference in the cumulative risk of primary outcome between early rhythm control and no rhythm control adherent to ABC pathway was evident (logrank: 0.099, $p=0.753$ ) (Fig. S1). Cox regression analysis confirmed no association between early rhythm control strategy vs. no rhythm control $\mathrm{ABC}$ adherent management in univariate (HR: $1.03,95 \%$ CI 0.71-1.49) or multivariable (HR: 0.99, 95\% CI 0.65-1.52) analyses.

\section{Discussion}

In this secondary analysis from the ESC-EHRA EORPAF General Long-Term Registry, only about one-third of European AF patients would be eligible for the original EAST-AFNET 4 study. Second, patients treated with early rhythm control were more likely to be younger, with less comorbidities, were more symptomatic and had a more established form of AF. Third, early rhythm control was associated with better quality of life during follow-up, but with an increased use of health-care resources, as well as a higher risk of hospital readmissions. Fourth, early rhythm control was associated with a lower rate of the composite 


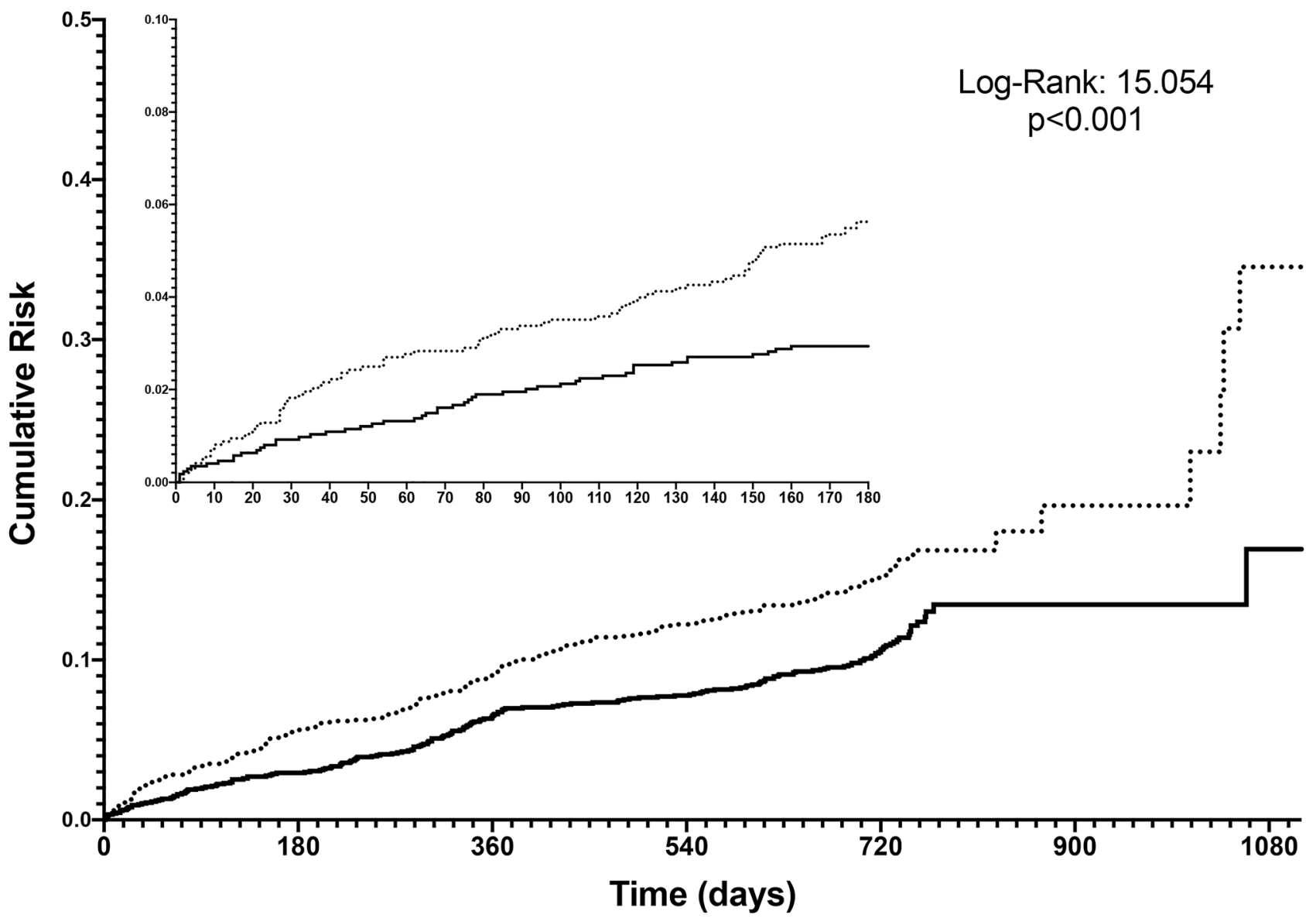

Fig. 2 Kaplan-Meier curves for EAST-AFNET 4 primary outcome. Black solid line $=$ early rhythm control; black dotted line $=$ no early rhythm control

Table 5 Cox regression analysis for main adverse events for those receiving early rhythm control versus no rhythm control

\begin{tabular}{llll}
\hline & $\begin{array}{l}\text { Univariate } \\
\text { HR [95\% CI] }\end{array}$ & $\begin{array}{l}\text { Model 1* } \\
\text { HR [95\% CI] }\end{array}$ & $\begin{array}{l}\text { Model 2 } \\
\text { HR [95\% CI }]\end{array}$ \\
\hline $\begin{array}{l}\text { EAST-AFNET } \\
\text { 4 primary } \\
\text { outcome }\end{array}$ & $0.69[0.57-0.83]$ & $0.83[0.68-1.01]$ & $0.84[0.66-1.19]$ \\
MACEs & $0.70[0.55-0.89]$ & $0.88[0.69-1.13]$ & $0.95[0.73-1.24]$ \\
All-cause death & $0.56[0.45-0.70]$ & $0.73[0.58-0.93]$ & $0.96[0.75-1.24]$ \\
\hline
\end{tabular}

$H R$ hazard ratio, MACEs major adverse cardiovascular events; for other acronyms please see previous tables' legends

*Adjusted for type of AF, EHRA score, $\mathrm{CHA}_{2} \mathrm{DS} 2$-VASc score, use of OAC

${ }^{\dagger}$ Adjusted for type of AF, EHRA score, age, sex, hypertension, diabetes mellitus, heart failure, severe coronary artery disease, valvular disease, left ventricular hypertrophy, peripheral artery disease, stroke/ transient ischaemic attack, chronic kidney disease, chronic obstructive pulmonary disease, malignancy, use of OAC primary outcome (CV death, any stroke, acute coronary syndrome or worsening heart failure). In the fully adjusted analyses, there were no statistical differences for the primary outcome, MACEs, and all-cause death for patients treated with early rhythm control (Graphical Abstract).

The debate comparing rate vs. rhythm control has been running for the last 20 years. In the 'Atrial Fibrillation Follow-Up Investigation of Rhythm Management' study, 4060 AF patients were randomized to rate vs. rhythm control strategies to establish if there were any differences in all-cause death. After 6 years of follow-up, this large trial did not find any differences in mortality between the two groups [22]. In the subsequent years, several other studies have shown a significant reduction of stroke risk and cardiovascular events, in patients managed with rhythm control strategies [23]. Conversely, other studies derived from observational registries report differences in event rates between patients treated with rate or rhythm control strategies, but these differences were no longer significant after adjustment for baseline characteristics [5, 24]. Several large meta-analyses have failed to show any significant 
differences in clinical outcomes between rate and rhythm control strategies [3, 4].

Other data from the 'Outcomes Registry for Better Informed Treatment of Atrial Fibrillation' and EORP-AF General Pilot registries report how in real-world clinical practice, rhythm control strategies are more commonly prescribed to younger and generally healthier patients, perhaps directly influencing the lower rate of events in multivariate analysis $[5,24]$. Nonetheless, the older trials were conducted $>2$ decades ago and, since then, there has been greater recognition and understanding of electromechanical substrate (and the possibility of early interventions to mitigate the so-called 'atrial cardiomyopathy' [25]) as well as the availability of improved (safer) drug therapies and catheter ablation approaches. Indeed, trial data regarding the use of catheter ablation in selected AF patients with heart failure show that using a rhythm control strategy may be associated with a lower rate of major adverse events [26, 27].

On this basis, the EAST-AFNET 4 trial investigators postulated that an early rhythm control could minimize AFinduced atrial damage, hence reducing the occurrence of clinical adverse events [20]. In this trial involving 135 centres, a total of 2789 patients were enrolled and randomized to receive early rhythm control or usual care and followed up for a median of 5.1 years. The early rhythm control arm was associated with a significant reduction in the composite primary outcome of CV death/stroke/hospitalization for heart failure or acute coronary syndrome (HR $0.79,95 \%$ CI 0.66-0.94), particularly driven by reduction in $\mathrm{CV}$ death and stroke [7].

In the current analysis of the EORP-AF registry, a large cohort of European AF patients, only one-third of patients could be considered for management with early rhythm control based on the EAST-AFNET 4 criteria. Among those patients qualifying for early rhythm control, such patients were younger and with less comorbidities, consistent with an early rhythm control intervention recommended to younger and healthier patients. While a difference in risk of major adverse outcomes exists when comparing early rhythm control to no rhythm control, this difference may be partly due to the differences in baseline characteristics, as shown in the fully adjusted model. Our analysis also shows that patients managed with early rhythm control are more commonly monitored and undergo further medical treatments, as underlined by the increased use of health-care resources and the higher risk for hospital readmission. Conversely, an early rhythm control strategy was associated with an overall better quality of life, consistent with previous data [3]. Data from the 'EdoxabaN vs. warfarin in subjectS UndeRgoing cardiovErsion of atrial fibrillation' trial show how receiving a cardioversion procedure was significantly associated with an improved patients' quality of life [28].
Indeed, early rhythm control as part of a structured follow-up regimen could partly explain the differences in clinical outcomes seen in the EAST-AFNET 4 trial, where the lower risk for the primary outcome could have been mediated by a better control and treatment of concomitant conditions [29-31]. Indeed, when directly comparing the early rhythm control group with the $\mathrm{ABC}$ pathway adherent patients, the absence of any difference in terms of primary outcome occurrence suggests that the beneficial effect of the early rhythm control could be attributed to a more comprehensive strategy of holistic care/management. Furthermore, the open label design in the EAST-AFNET-4 trial could have influenced recurring (and more common) medical checks.

Moreover, the higher incidence rates of the primary outcome both in patients with no rhythm control and early rhythm control, compared to the original study cohort's event rates, underlines how our real-life cohort is significantly at higher risk for outcomes compared to that seen in the selected population included within a randomized clinical trial. While this poses some limitations to the generalizability of EAST-AFNET 4 trial in real-life patients, it underlines that general AF patients have strong burden of risk related to the complexity of their overall clinical features, questioning whether the 'one intervention fits all' application of an early rhythm control approach irrespective of the clinical presentation is appropriate. Also, this given greater justification for integrated or holistic management of AF patients, consistent with the ESC AF guideline-recommended $\mathrm{ABC}$ pathway [1].

Indeed, the use of a rhythm control intervention could be prioritized for those patients with a significant burden of symptoms, as part of a comprehensive management of $\mathrm{AF}$ patients. The use of rate and rhythm control to reduce the burden of symptoms is one of the pillars of the $\mathrm{ABC}$ pathway [1]. Such a structured approach to holistic AF care, including proactive risk evaluation and management, has been shown to be associated with improved clinical outcomes, especially with a reduction in hospitalizations and clinical events [19, 32-34].

\section{Limitations}

This analysis has some limitations. Being a secondary analysis of an observational registry, the original study design was not powered to examine differences in specific subgroups and for specific rhythm strategies. Since a higher burden of symptoms was associated with use of early rhythm control, we could hypothesize that this could represent a bias influencing the use of health-care resources. The inclusion of EHRA score as covariate in the regression analyses regarding the use of health-care resources would minimize such bias. 
Despite the use of adjusted analyses, differences in baseline characteristics could have significantly influenced our results, especially with progressive ageing and incident comorbidities [35-38]. Finally, the non-randomized treatment allocation of patients to the groups examined clearly limits the generalizability of our conclusions, raising the need of interpreting our data with caution. Furthermore, while we fully recognize that observational data are no substitute for clinical trials, we analysed a real-world European cohort that is nearly twofold in size compared to the original EAST-AFNET 4 trial, reflecting the generalizability of our results rather than the selective inclusion/exclusion criteria of trial cohorts.

\section{Conclusions}

In this secondary analysis of a large contemporary cohort of AF patients, only one-third of patients would have been eligible for inclusion into the EAST-AFNET 4 trial. Use of an early rhythm control strategy was associated with a lower rate of major adverse events, but this difference was non-significant on multivariate analysis, being mediated by differences in baseline characteristics and clinical risk profile. Early rhythm control was associated with a higher use of health-care resources and risk of hospital admission, despite showing better quality of life.

Supplementary Information The online version contains supplementary material available at https://doi.org/10.1007/s00392-021-01914-y.

Acknowledgements The authors acknowledge EORP Oversight Committee, Executive and Steering Committees (National Coordinators) of the EURObservational Research Programme (EORP)—Atrial Fibrillation General Long-Term (EORP-AF Gen LT) Registry of the European Society of Cardiology (ESC). Data collection was conducted by the EORP department by Patti-Ann McNeill as Project Officer and Viviane Missiamenou as Data Manager. Statistical analyses were performed by Cécile Laroche. Overall activities were coordinated and supervised by Doctor Aldo P. Maggioni (EORP Scientific Coordinator). The members of the ESC-EHRA EORP-AF Long-Term General Registry Investigators are: Executive Committee: G. Boriani (Chair), G.Y.H. Lip, L. Tavazzi, A. P. Maggioni, G-A. Dan, T. Potpara, M. Nabauer, F. Marin, Z. Kalarus, L. Fauchier, R. Ferrari, A. Shantsila. Steering Committee (National Coordinators): A. Goda, G. Mairesse, T.Shalganov, L. Antoniades, M. Taborsky, S. Riahi, P. Muda, I. García Bolao, O. Piot, M. Nabauer, K. Etsadashvili, E.Simantirakis, M. Haim, A. Azhari, J. Najafian, M. Santini, E. Mirrakhimov, K.a Kulzida, A. Erglis, L. Poposka, M. Burg, H. Crijns, Ö. Erküner, D. Atar, R. Lenarczyk, M. Martins Oliveira, D. Shah, G-A. Dan, E. Serdechnaya, T. Potpara, E. Diker, G.Y.H. Lip, D. Lane. Investigators: ALBANIA Durrës: E. Zëra, Tirana: U. Ekmekçiu, V. Paparisto, M. Tase, Tirana: H. Gjergo, J. Dragoti, A. Goda, BELGIUM Bastogne: M. Ciutea, N. Ahadi, Z. el Husseini, M. Raepers, Gilly: J. Leroy, P. Haushan, A. Jourdan, Haine Saint Paul: C. Lepiece, Hasselt: L. Desteghe, J. Vijgen, P. Koopman, G. Van Genechten, H. Heidbuchel, Kortrijk: T. Boussy, M. De Coninck, H. Van Eeckhoutte, N. Bouckaert, La Louviere: A. Friart, J. Boreux, C. Arend, Liege: P. Evrard, Liège: L. Stefan, E. Hoffer, J. Herzet, M.
Massoz, Liège: C. Celentano, M. Sprynger, L. Pierard, Liège: P. Melon, Overpelt: B. Van Hauwaert, C. Kuppens, D. Faes, D. Van Lier, A. Van Dorpe, Waremme: A. Gerardy, Yvoir: O. Deceuninck, O. Xhaet, F. Dormal, E. Ballant, D. Blommaert, BULGARIA Pleven: D. Yakova, M. Hristov, T. Yncheva, N. Stancheva, S. Tisheva, Plovdiv: M. Tokmakova, F. Nikolov, D. Gencheva, Sofia: T. Shalganov, B. Kunev, M. Stoyanov, Sofia: D. Marchov, V. Gelev, V. Traykov, Varna: A. Kisheva, H. Tsvyatkov, R. Shtereva, S. Bakalska-Georgieva, S. Slavcheva, Y. Yotov, CZECH REPUBLIC Ústí nad Labem: M. Kubíčková, DENMARK Aalborg: A. Marni Joensen, A. Gammelmark, L. Hvilsted Rasmussen, P. Dinesen, S. Riahi, S. Krogh Ven $\varnothing$, B. Sorensen, A. Korsgaard, K. Andersen, C. Fragtrup Hellum, Esbjerg: A. Svenningsen, O. Nyvad, P. Wiggers, Herning: O. May, A. Aarup, B. Graversen, L. Jensen, M. Andersen, M. Svejgaard, S. Vester, S. Hansen, V. Lynggaard, Madrid: M. Ciudad, Tallinn: R. Vettus, Tartu: P. Muda, ESTONIA Elche, Alicante: A. Maestre, Toledo: S. Castaño, FRANCE Abbeville: S. Cheggour, Abbeville: J. Poulard, V. Mouquet, S. Leparrée, Aix-en-Provence: J. Bouet, J. Taieb, Amiens: A. Doucy, H. Duquenne, Angers: A. Furber, J. Dupuis, J. Rautureau, Aurillac: M. Font, P. Damiano, Avignon Cedex: M. Lacrimini, Brest: J. Abalea, S. Boismal, T. Menez, J. Mansourati, Chartres: G. Range, H. Gorka, C. Laure, C. Vassalière, Creteil: N. Elbaz, N. Lellouche, K. Djouadi, Montpellier: F. Roubille, D. Dietz, J. Davy, Nimes: M. Granier, P. Winum, C. Leperchois-Jacquey, Paris: H. Kassim, E. Marijon, J. Le Heuzey, Paris: J. Fedida, C. Maupain, C. Himbert, E. Gandjbakhch, F. Hidden-Lucet, G. Duthoit, N. Badenco, T. Chastre, X. Waintraub, M. Oudihat, J. Lacoste, C. Stephan, Pau: H. Bader, N. Delarche, L. Giry, Pessac: D. Arnaud, C. Lopez, F. Boury, I. Brunello, M. Lefèvre, R. Mingam, M. Haissaguerre, Rennes: M. Le Bidan, D. Pavin, V. Le Moal, C. Leclercq, Saint Denis: O. Piot, T. Beitar, Saint Etienne: I. Martel, A. Schmid, N. Sadki, C. Romeyer-Bouchard, A. Da Costa, Tours: I. Arnault, M. Boyer, C. Piat, L. Fauchier, FYR MACEDONIA Bitola: N. Lozance, S. Nastevska, Ohrid: A. Doneva, B. Fortomaroska Milevska, B. Sheshoski, K. Petroska, N. Taneska, N. Bakrecheski, Skopje: K. Lazarovska, S. Jovevska, V. Ristovski, A. Antovski, Skopje: E. Lazarova, I. Kotlar, J. Taleski, L. Poposka, S. Kedev, Skopje: N. Zlatanovik, Stip: S. Jordanova, T. Bajraktarova Proseva, S. Doncovska, GEORGIA Tbilisi: D. Maisuradze, A. Esakia, E. Sagirashvili, K. Lartsuliani, N. Natelashvili, N. Gumberidze, R. Gvenetadze, Tbilisi: K. Etsadashvili, N. Gotonelia, N. Kuridze, Tbilisi: G. Papiashvili, I. Menabde, GERMANY Aachen: S. Glöggler, A. Napp, C. Lebherz, H. Romero, K. Schmitz, M. Berger, M. Zink, S. Köster, J. Sachse, E. Vonderhagen, G. Soiron, K. Mischke, Bad Reichenhall: R. Reith, M. Schneider, Berlin: W. Rieker, Biberach: D. Boscher, A. Taschareck, A. Beer, Boppard: D. Oster, Brandenburg: O. Ritter, J. Adamczewski, S. Walter, Chemnitz: A. Frommhold, E. Luckner, J. Richter, M. Schellner, S. Landgraf, S. Bartholome, Chemnitz: R. Naumann, J. Schoeler, Dachau: D. Westermeier, F. William, K. Wilhelm, M. Maerkl, Detmold: R. Oekinghaus, M. Denart, M. Kriete, U. Tebbe, Ebersbach: T. Scheibner, Erlangen: M. Gruber, A. Gerlach, C. Beckendorf, L. Anneken, M. Arnold, S. Lengerer, Z. Bal, C. Uecker, H. Förtsch, S. Fechner, V. Mages, Friedberg: E. Martens, H. Methe, Göttingen: T. Schmidt, Hamburg: B. Schaeffer, B. Hoffmann, J. Moser, K. Heitmann, S. Willems, S. Willems, Hartmannsdorf: C. Klaus, I. Lange, Heidelberg: M. Durak, E. Esen, Itzehoe: F. Mibach, H. Mibach, Kassel: A. Utech, Kirchzarten: M. Gabelmann, R. Stumm, V. Ländle, Koblenz: C. Gartner, C. Goerg, N. Kaul, S. Messer, D. Burkhardt, C. Sander, R. Orthen, S. Kaes, Köln: A. Baumer, F. Dodos, Königsbrück: A. Barth, G. Schaeffer, Leisnig: J. Gaertner, J. Winkler, Leverkusen: A. Fahrig, J. Aring, I. Wenzel, Limburg: S. Steiner, A. Kliesch, E. Kratz, K. Winter, P. Schneider, Ludwigsburg: A. Haag, I. Mutscher, R. Bosch, Markkleeberg: J. Taggeselle, S. Meixner, Meissen: A. Schnabel, Meppen: A. Shamalla, H. Hötz, A. Korinth, Merzig: C. Rheinert, Moosburg: G. Mehltretter, Mühldorf: B. Schön, N. Schön, A. Starflinger, E. Englmann, Munich: G. Baytok, T. Laschinger, G. Ritscher, Munich: A. Gerth, Münster: D. Dechering, L. Eckardt, Nienburg: M. Kuhlmann, 
N. Proskynitopoulos, Paderborn: J. Brunn, K. Foth, Pirna: C. Axthelm, H. Hohensee, K. Eberhard, S. Turbanisch, Plauen: N. Hassler, A. Koestler, Riesa: G. Stenzel, Riesa: D. Kschiwan, M. Schwefer, S. Neiner, S. Hettwer, Rotenburg a.d. Fulda: M. Haeussler-Schuchardt, R. Degenhardt, S. Sennhenn, S. Steiner, Starnberg: M. Brendel, Westerstede: A. Stoehr, W. Widjaja, S. Loehndorf, A. Logemann, J. Hoskamp, J. Grundt, Zorneding: M. Block, Zwiesel: R. Ulrych, A. Reithmeier, V. Panagopoulos, ITALY Bologna: C. Martignani, D. Bernucci, E. Fantecchi, I. Diemberger, M. Ziacchi, M. Biffi, P. Cimaglia, J. Frisoni, G. Boriani, Firenze: I. Giannini, S. Boni, S. Fumagalli, S. Pupo, A. Di Chiara, P. Mirone, Modena: E. Fantecchi, G. Boriani, F. Pesce, C. Zoccali, V.L. Malavasi, KAZAKHSTAN Almaty: A. Mussagaliyeva, B. Ahyt, Z. Salihova, K. Koshum-Bayeva, KYRGYZSTAN Bishkek: A. Kerimkulova, A. Bairamukova, E. Mirrakhimov, LATVIA Riga: B. Lurina, R. Zuzans, S. Jegere, I. Mintale, K. Kupics, K. Jubele, A. Erglis, O. Kalejs, MALTA Birkirkara: K. Vanhear, M. Burg, M. Cachia, E. Abela, S. Warwicker, T. Tabone, R. Xuereb, MONTENEGRO Podgorica: D. Asanovic, D. Drakalovic, M. Vukmirovic, N. Pavlovic, L. Music, N. Bulatovic, A. Boskovic, NETHERLANDS Almere: H. Uiterwaal, N. Bijsterveld, Amsterdam: J. De Groot, J. Neefs, N. van den Berg, F. Piersma, A. Wilde, Delfzijl: V. Hagens, Enschede: J. Van Es, J. Van Opstal, B. Van Rennes, H. Verheij, W. Breukers, Heerenveen: G. Tjeerdsma, R. Nijmeijer, D. Wegink, R. Binnema, Hengelo: S. Said, Maastricht: Ö. Erküner, S. Philippens, W. van Doorn, H. Crijns, Rotterdam: T. Szili-Torok, R. Bhagwandien, P. Janse, A. Muskens, s-Hertogenbosch: M. van Eck, R. Gevers, N. van der Ven, Venlo: A. Duygun, B. Rahel, J. Meeder, NORWAY Oslo: A. Vold, C. Holst Hansen, I. Engset, D. Atar, POLAND Bytom: B. Dyduch-Fejklowicz, E. Koba, M. Cichocka, Cieszyn: A. Sokal, A. Kubicius, E. Pruchniewicz, Gliwice: A. Kowalik-Sztylc, W. Czapla, Katowice: I. Mróz, M. Kozlowski, T. Pawlowski, M. Tendera, Katowice: A. Winiarska-Filipek, A. Fidyk, A. Slowikowski, M. Haberka, M. Lachor-Broda, M. Biedron, Z. Gasior, Kielce: M. Kołodziej, M. Janion, Kielce: I. Gorczyca-Michta, B. Wozakowska-Kaplon, Łódź: M. Stasiak, P. Jakubowski, T. Ciurus, J. Drozdz, Łódź: M. Simiera, P. Zajac, T. Wcislo, P. Zycinski, J. Kasprzak, Nysa: A. Olejnik, E. Harc-Dyl, J. Miarka, M. Pasieka, M. Ziemińska-Łuć, W. Bujak, Opoczno: A. Śliwiński, A. Grech, J. Morka, K. Petrykowska, M. Prasał, Opole: G. Hordyński, P. Feusette, P. Lipski, A. Wester, Radlin: W. Streb, Rzeszów: J. Romanek, P. Woźniak, M. Chlebuś, P. Szafarz, W. Stanik, Szczecin: M. Zakrzewski, J. Kaźmierczak, Szczecin: A. Przybylska, E. Skorek, H. Błaszczyk, M. Stępień, S. Szabowski, W. Krysiak, M. Szymańska, Tarnów: J. Karasiński, J. Blicharz, M. Skura, Warsaw: K. Hałas, L. Michalczyk, Z. Orski, K. Krzyżanowski, A. Skrobowski, Warsaw: L. Zieliński, M. Tomaszewska-Kiecana, M. Dłużniewski, Warsaw: M. Kiliszek, M. Peller, M. Budnik, P. Balsam, G. Opolski, A. Tymińska, K. Ozierański, A. Wancerz, Warsaw: A. Borowiec, E. Majos, R. Dabrowski, H. Szwed, Zabrze: A. MusialikLydka, Zabrze: A. Leopold-Jadczyk, E. Jedrzejczyk-Patej, M. Koziel, R. Lenarczyk, M. Mazurek, Z. Kalarus, Zabrze: K. Krzemien-Wolska, P. Starosta, E. Nowalany-Kozielska, Zakopane: A. Orzechowska, M. Szpot, M. Staszel, PORTUGAL Almada: S. Almeida, H. Pereira, L. Brandão Alves, R. Miranda, L. Ribeiro, Carnaxide Lisboa: F. Costa, F. Morgado, P. Carmo, P. Galvao Santos, R. Bernardo, P. Adragão, Santarém: G. Ferreira da Silva, M. Peres, M. Alves, M. Leal, Vila Real: A. Cordeiro, P. Magalhães, P. Fontes, S. Leão, Viseu: A. Delgado, A. Costa, B. Marmelo, B. Rodrigues, D. Moreira, J. Santos, L. Santos, ROMANIA Arad: A. Terchet, D. Darabantiu, S. Mercea, V. Turcin Halka, A. Pop Moldovan, Brasov: A. Gabor, B. Doka, G. Catanescu, H. Rus, L. Oboroceanu, E. Bobescu, Bucharest: R. Popescu, A. Dan, A. Buzea, I. Daha, G. Dan, I. Neuhoff, Bucharest: M. Baluta, R. Ploesteanu, N. Dumitrache, M. Vintila,Bucharest: A. Daraban, C. Japie, E. Badila, H. Tewelde, M. Hostiuc, S. Frunza, E. Tintea, D. Bartos, Bucharest: A. Ciobanu, I. Popescu, N. Toma, C. Gherghinescu, D. Cretu, N. Patrascu, C. Stoicescu, C. Udroiu, G. Bicescu, V. Vintila, D. Vinereanu, M. Cinteza, R. Rimbas, Iași: M. Grecu, Oradea: A. Cozma, F. Boros, M. Ille, O. Tica, R. Tor, A. Corina, A. Jeewooth, B. Maria,
C. Georgiana, C. Natalia, D. Alin, D. Dinu-Andrei, M. Livia, R. Daniela, R. Larisa, S. Umaar, T. Tamara, M. Ioachim Popescu, Târgu Mureș: D. Nistor, I. Sus, O. Coborosanu, Timișoara: N. Alina-Ramona, R. Dan, L. Petrescu, Timișoara: G. Ionescu, I. Popescu, C. Vacarescu, E. Goanta, M. Mangea, A. Ionac, C. Mornos, D. Cozma, S. Pescariu, RUSSIAN FEDERATION Arkhangelsk: E. Solodovnicova, I. Soldatova, J. Shutova, L. Tjuleneva, T. Zubova, V. Uskov, Arkhangelsk: D. Obukhov, G. Rusanova, Arkhangelsk: I. Soldatova, N. Isakova, S. Odinsova, T. Arhipova, Arkhangelsk: E. Kazakevich, E. Serdechnaya, O. Zavyalova, Saint-Petersburg: T. Novikova, Saint-Petersburg: I. Riabaia, S. Zhigalov, Saint-Petersburg: E. Drozdova, I. Luchkina, Y. Monogarova, Vladivostok: D. Hegya, L. Rodionova, L. Rodionova, V. Nevzorova, Vladivostok: I. Soldatova, O. Lusanova, SERBIA Belgrade: A. Arandjelovic, D. Toncev, M. Milanov, N. Sekularac, Belgrade: M. Zdravkovic, S. Hinic, S. Dimkovic, T. Acimovic, J. Saric, Belgrade: M. Polovina, T. Potpara, B. Vujisic-Tesic, M. Nedeljkovic, Belgrade: M. Zlatar, M. Asanin, Belgrade: V. Vasic, Z. Popovic, Belgrade: D. Djikic, M. Sipic, V. Peric, B. Dejanovic, N. Milosevic, Belgrade: A. Stevanovic, A. Andric, B. Pencic, M. Pavlovic-Kleut, V. Celic, Kragujevac: M. Pavlovic, M. Petrovic, M. Vuleta, N. Petrovic, S. Simovic, Z. Savovic, S. Milanov, G. Davidovic, V. Iric-Cupic, Niška Banja: D. Simonovic, M. Stojanovic, S. Stojanovic, V. Mitic, V. Ilic, D. Petrovic, M. Deljanin Ilic, S. Ilic, V. Stoickov, Pirot: S. Markovic, Šabac:S. Kovacevic. SPAIN Alicante: A. García Fernandez, Benalmadena: A. Perez Cabeza, Córdoba: M. Anguita, Granada: L. Tercedor Sanchez, Huarte: E. Mau, J. Loayssa, M. Ayarra, M. Carpintero, Madrid: I. Roldán Rabadan, Murcia: M. Leal, Murcia: M. Gil Ortega, Murcia: A. Tello Montoliu, E. Orenes Piñero, S. Manzano Fernández, F. Marín, A. Romero Aniorte, A. Veliz Martínez, M. Quintana Giner, Pamplona: G. Ballesteros, M. Palacio, O. Alcalde, I. García-Bolao, San Juan de Alicante: V. Bertomeu Gonzalez, Santiago de Compostela: F. Otero-Raviña, J. García Seara, J. Gonzalez Juanatey, SWITZERLAND Geneva: N. Dayal, P. Maziarski, P. Gentil-Baron, D. Shah, TURKEY Adana: M. Koç, Afyon: E. Onrat, I. E. Dural, Ankara: K. Yilmaz, B. Özin, Ankara: S. Tan Kurklu, Y. Atmaca, Ankara: U. Canpolat, L. Tokgozoglu, Ankara: A. K. Dolu, B. Demirtas, D. Sahin, Ankara: O. Ozcan Celebi, E. Diker, Antalya: G. Gagirci, Bayraklı/Izmir: U.O.Turk, Bursa: H. Ari, Diyarbakır: N. Polat, N. Toprak, Gaziantep: M. Sucu, Görükle-Bursa: O. Akin Serdar, Istanbul: A. Taha Alper, Istanbul: A. Kepez, Istanbul: Y. Yuksel, Kurupelit-Samsun: A. Uzunselvi, S. Yuksel, M. Sahin, Merkez/Düzce: O. Kayapinar, Mersin: T. Ozcan, Sivas: H. Kaya, M. B. Yilmaz, Trabzon: M. Kutlu, Yüreğir-Adana: M. Demir, UNITED KINGDOM Barnstaple: C. Gibbs, S. Kaminskiene, M. Bryce, A. Skinner, G. Belcher, J. Hunt, L. Stancombe, B. Holbrook, C. Peters, S. Tettersell, Birmingham: A. Shantsila, D. Lane, K. Senoo, M. Proietti, K. Russell, P. Domingos, S. Hussain, J. Partridge, R. Haynes, S. Bahadur, R. Brown, S. McMahon, G. Y H Lip, Blackburn: J. McDonald, K. Balachandran, R. Singh, S. Garg, H. Desai, K. Davies, W. Goddard, Blackpool: G. Galasko, I. Rahman, Y. Chua, O. Payne, S. Preston, O. Brennan, L. Pedley, C. Whiteside, C. Dickinson, J. Brown, K. Jones, L. Benham, R. Brady, Carlisle: L. Buchanan, A. Ashton, H. Crowther, H. Fairlamb, S. Thornthwaite, C. Relph, A. McSkeane, U. Poultney, N. Kelsall, P. Rice, T. Wilson, Chertsey: M. Wrigley, R. Kaba, T. Patel, E. Young, J. Law, Cramlington: C. Runnett, H. Thomas, H. McKie, J. Fuller, S. Pick, Exeter: A. Sharp, A. Hunt, K. Thorpe, C. Hardman, E. Cusack, L. Adams, M. Hough, S. Keenan, A. Bowring, J. Watts, Great Yarmouth: J. Zaman, K. Goffin, H. Nutt, Harrogate: Y. Beerachee, J. Featherstone, C. Mills, J. Pearson, L. Stephenson, Huddersfield: S. Grant, A. Wilson, C. Hawksworth, I. Alam, M. Robinson, S. Ryan, Macclesfield: R. Egdell, E. Gibson, M. Holland, D. Leonard, Maidstone: B. Mishra, S. Ahmad, H. Randall, J. Hill, L. Reid, M. George, S. McKinley, L. Brockway, W. Milligan, Manchester: J. Sobolewska, J. Muir, L. Tuckis, L. Winstanley, P. Jacob, S. Kaye, L. Morby, Nottingham: A. Jan, T. Sewell, Poole: C. Boos, B. Wadams, C. Cope, P. Jefferey, Portsmouth: N. Andrews, A. Getty, A. Suttling, C. Turner, K. Hudson, R. Austin, S. Howe, Redhill: R. Iqbal, N. Gandhi, 
K. Brophy, P. Mirza, E. Willard, S. Collins, N. Ndlovu, Rhyl: E. Subkovas, V. Karthikeyan, L. Waggett, A. Wood, A. Bolger, J. Stockport, L. Evans, E. Harman, J. Starling, L. Williams, V. Saul, Salisbury: M. Sinha, L. Bell, S. Tudgay, S. Kemp, J. Brown, L. Frost, Shrewsbury: T. Ingram, A. Loughlin, C. Adams, M. Adams, F. Hurford, C. Owen, C. Miller, D. Donaldson, H. Tivenan, H. Button, South Shields: A. Nasser, O. Jhagra, B. Stidolph, C. Brown, C. Livingstone, M. Duffy, P. Madgwick, Southampton: P. Roberts, E. Greenwood, L. Fletcher, M. Beveridge, S. Earles, Taunton: D. McKenzie, D. Beacock, M. Dayer, M. Seddon, D. Greenwell, F. Luxton, F. Venn, H. Mills, J. Rewbury, K. James, K. Roberts, L. Tonks, Torquay: D. Felmeden, W. Taggu, A. Summerhayes, D. Hughes, J. Sutton, L. Felmeden, Watford: M. Khan, E. Walker, L. Norris, L. O'Donohoe, Weston-super-Mare: A. Mozid, H. Dymond, H. Lloyd-Jones, G. Saunders, D. Simmons, D. Coles, D. Cotterill, S. Beech, S. Kidd, Wolverhampton: B. Wrigley, S. Petkar, A. Smallwood, R. Jones, E. Radford, S. Milgate, S. Metherell, V. Cottam, Yeovil: C. Buckley, A. Broadley, D. Wood, J. Allison, K. Rennie, L. Balian, L. Howard, L. Pippard, S. Board, T. Pitt-Kerby.

Funding Open access funding provided by Università degli Studi di Milano within the CRUI-CARE Agreement. Since the start of EORP, the following companies have supported the programme: Abbott Vascular Int. (2011-2021), Amgen Cardiovascular (2009-2018), AstraZeneca (2014-2021), Bayer (2009-2018), Boehringer Ingelheim (2009-2019), Boston Scientific (2009-2012), The Bristol Myers Squibb and Pfizer Alliance (2011-2016), The Alliance Daiichi Sankyo Europe GmbH and Eli Lilly and Company (2011-2017), Edwards (2016-2019), Gedeon Richter Plc. (2014-2017), Menarini Int. Op. (2009-2012), MSD-Merck \& Co. (2011-2014), Novartis Pharma AG (2014-2020), ResMed (2014-2016), Sanofi (2009-2011), SERVIER (2010-2021), Vifor (2019-2022).

\section{Declarations}

Conflict of interest DL has received investigator-initiated educational grants from Bristol-Myers Squibb (BMS), has been a speaker for Boehringer Ingelheim and BMS/Pfizer and has consulted for BMS, Boehringer Ingelheim, and Daiichi-Sankyo. LF has been consultant or speaker for Bayer, BMS/Pfizer, Boehringer Ingelheim, Medtronic and Novartis; GB received small speaker's fees from Medtronic, Boston, Boehringer Ingelheim and Bayer; GYHL has been consultant and speaker for BMS/Pfizer, Boehringer Ingelheim and Daiichi-Sankyo. No fees were received personally. All the disclosures happened outside the submitted work. All other authors have nothing to declare.

Open Access This article is licensed under a Creative Commons Attribution 4.0 International License, which permits use, sharing, adaptation, distribution and reproduction in any medium or format, as long as you give appropriate credit to the original author(s) and the source, provide a link to the Creative Commons licence, and indicate if changes were made. The images or other third party material in this article are included in the article's Creative Commons licence, unless indicated otherwise in a credit line to the material. If material is not included in the article's Creative Commons licence and your intended use is not permitted by statutory regulation or exceeds the permitted use, you will need to obtain permission directly from the copyright holder. To view a copy of this licence, visit http://creativecommons.org/licenses/by/4.0/.

\section{References}

1. Hindricks G, Potpara T, Dagres N et al (2021) 2020 ESC Guidelines for the diagnosis and management of atrial fibrillation developed in collaboration with the European Association for Cardio-Thoracic Surgery (EACTS). Eur Heart J 42:373-498. https://doi.org/10.1093/eurheartj/ehaa612

2. Lip GYH (2017) The ABC pathway: an integrated approach to improve AF management. Nat Rev Cardiol 14:627-628. https:// doi.org/10.1038/nrcardio.2017.153

3. Al-Khatib SM, LaPointe NMA, Chatterjee R et al (2014) Rateand rhythm-control therapies in patients with atrial fibrillation: a systematic review. Ann Intern Med 160:760-773. https://doi.org/ 10.7326/M13-1467

4. Sethi NJ, Feinberg J, Nielsen EE et al (2017) The effects of rhythm control strategies versus rate control strategies for atrial fibrillation and atrial flutter: a systematic review with meta-analysis and Trial Sequential Analysis. PLoS One 12:e0186856. https://doi. org/10.1371/journal.pone.0186856

5. Purmah Y, Proietti M, Laroche C et al (2018) Rate vs. rhythm control and adverse outcomes among European patients with atrial fibrillation. Europace 20:243-252. https://doi.org/10.1093/europ ace/euw421

6. Van Gelder IC, Rienstra M, Crijns HJGM, Olshansky B (2016) Rate control in atrial fibrillation. Lancet 388:818-828. https://doi. org/10.1016/S0140-6736(16)31258-2

7. Kirchhof P, Camm AJ, Goette A et al (2020) Early rhythmcontrol therapy in patients with atrial fibrillation. N Engl J Med 383:1305-1316. https://doi.org/10.1056/nejmoa2019422

8. Lip GYH, Laroche C, Dan G-A et al (2014) A prospective survey in European Society of Cardiology member countries of atrial fibrillation management: baseline results of EURObservational Research Programme Atrial Fibrillation (EORP-AF) Pilot General Registry. Europace 16:308-319. https://doi.org/10.1093/europace/ eut373

9. Lip GYH, Laroche C, Ioachim PM et al (2014) Prognosis and treatment of atrial fibrillation patients by European cardiologists: one year follow-up of the EURObservational Research Programme-Atrial Fibrillation General Registry Pilot Phase (EORPAF Pilot registry). Eur Heart J 35:3365-3376. https://doi.org/10. 1093/eurheartj/ehu374

10. Proietti M, Laroche C, Opolski G et al (2017) "Real-world" atrial fibrillation management in Europe: observations from the 2-year follow-up of the EURObservational Research Programme-Atrial Fibrillation General Registry Pilot Phase. Europace 19:722-733. https://doi.org/10.1093/europace/euw112

11. Boriani G, Proietti M, Laroche $C$ et al (2018) Changes to oral anticoagulant therapy and risk of death over a 3-year follow-up of a contemporary cohort of European patients with atrial fibrillation final report of the EURObservational Research Programme on Atrial Fibrillation (EORP-AF) pilot general registry. Int J Cardiol 271:68-74. https://doi.org/10.1016/j.ijcard.2018.05.034

12. Boriani G, Proietti M, Laroche C et al (2018) Contemporary stroke prevention strategies in 11096 European patients with atrial fibrillation: a report from the EURObservational Research Programme on Atrial Fibrillation (EORP-AF) Long-Term General Registry. Europace 20:747-757. https://doi.org/10.1093/europace/eux301

13. Boriani G, Proietti M, Laroche $C$ et al (2019) Association between antithrombotic treatment and outcomes at 1-year follow-up in patients with atrial fibrillation: the EORP-AF General Long-Term Registry. Europace 21:1013-1022. https://doi.org/10.1093/europ ace/euz032

14. Lip GYH, Nieuwlaat R, Pisters R et al (2010) Refining clinical risk stratification for predicting stroke and thromboembolism in atrial fibrillation using a novel risk factor-based approach: the euro heart survey on atrial fibrillation. Chest 137:263-272. https://doi. org/10.1378/chest.09-1584

15. Pisters R, Lane DA, Nieuwlaat $R$ et al (2010) A novel user-friendly score (HAS-BLED) to assess 1-year risk of major bleeding in 
patients with atrial fibrillation: the Euro Heart Survey. Chest 138:1093-1100. https://doi.org/10.1378/chest.10-0134

16. Rockwood K, Mitnitski A (2007) Frailty in relation to the accumulation of deficits. J Gerontol Ser A Biol Sci Med Sci 62:722-727

17. Masnoon N, Shakib S, Kalisch-Ellett L, Caughey GE (2017) What is polypharmacy? A systematic review of definitions. BMC Geriatr 17:230. https://doi.org/10.1186/s12877-017-0621-2

18. Proietti M, Lip GYH, Laroche C et al (2021) Relation of outcomes to ABC (Atrial Fibrillation Better Care) pathway adherent care in European patients with atrial fibrillation: an analysis from the ESC-EHRA EORP Atrial Fibrillation General Long-Term (AFGen LT) Registry. Europace 23:174-183. https://doi.org/10. 1093/europace/euaa274

19. Romiti GF, Pastori D, Rivera-Caravaca JM et al (2021) Adherence to the 'atrial fibrillation better care' pathway in patients with atrial fibrillation: impact on clinical outcomes-a systematic review and meta-analysis of 285,000 patients. Thromb Haemost. https://doi. org/10.1055/a-1515-9630

20. Kirchhof P, Breithardt G, Camm AJ et al (2013) Improving outcomes in patients with atrial fibrillation: rationale and design of the early treatment of atrial fibrillation for stroke prevention trial. Am Heart J 166:442-448. https://doi.org/10.1016/j.ahj.2013.05. 015

21. Dudink EAMP, Erküner Ö, Berg J et al (2018) The influence of progression of atrial fibrillation on quality of life: a report from the Euro Heart Survey. Europace 20:929-934. https://doi.org/10. 1093/europace/eux217

22. Wyse DG, Waldo AL, DiMarco JP et al (2002) A comparison of rate control and rhythm control in patients with atrial fibrillation. N Engl J Med 347:1825-1833. https://doi.org/10.1056/NEJMo a021328

23. Tsadok MA, Jackevicius CA, Essebag V et al (2012) Rhythm versus rate control therapy and subsequent stroke or transient ischemic attack in patients with atrial fibrillation. Circulation 126:2680-2687. https://doi.org/10.1161/CIRCULATIONAHA. 112.092494

24. Noheria A, Shrader P, Piccini JP et al (2016) Rhythm control versus rate control and clinical outcomes in patients with atrial fibrillation. JACC Clin Electrophysiol 2:221-229. https://doi.org/ 10.1016/j.jacep.2015.11.001

25. Goette A, Kalman JM, Aguinaga L et al (2016) EHRA/HRS/ APHRS/SOLAECE expert consensus on atrial cardiomyopathies: definition, characterization, and clinical implication. Europace 18:1455-1490. https://doi.org/10.1093/europace/euw161

26. Marrouche NF, Brachmann J, Andresen D et al (2018) Catheter ablation for atrial fibrillation with heart failure. N Engl J Med 378:417-427. https://doi.org/10.1056/NEJMoa1707855

27. Asad ZUA, Yousif A, Khan MS et al (2019) Catheter ablation versus medical therapy for atrial fibrillation: a systematic review and meta-analysis of randomized controlled trials. Circ Arrhythmia Electrophysiol. https://doi.org/10.1161/CIRCEP.119.007414

28. Goette A, Kwong WJ, Ezekowitz MD et al (2018) Edoxaban therapy increases treatment satisfaction and reduces utilization of healthcare resources: an analysis from the EdoxabaN vs. Warfarin in subjects UndeRgoing cardioversion of atrial fibrillation (ENSURE-AF) study. Europace 20:1936-1943. https://doi.org/ 10.1093/europace/euy141

29. Galiuto L, Patrono C (2020) Early rhythm control for early atrial fibrillation? Comment on the EAST-AFNET 4 trial. Eur Heart J 41:3987-3988. https://doi.org/10.1093/eurheartj/ehaa812

30. Yang E, Tang O, Metkus T et al (2020) The role of timing in treatment of atrial fibrillation: an AFFIRM substudy. Heart Rhythm. https://doi.org/10.1016/j.hrthm.2020.12.025

31. Bunch TJ, Steinberg BA (2020) Revisiting rate versus rhythm control in atrial fibrillation - timing matters. N Engl J Med 383:1383-1384. https://doi.org/10.1056/nejme2027180

32. Guo Y, Guo J, Shi X et al (2020) Mobile health technology-supported atrial fibrillation screening and integrated care: a report from the mAFA-II trial long-term extension cohort. Eur J Intern Med 82:105-111. https://doi.org/10.1016/j.ejim.2020.09.024

33. Guo Y, Lane DA, Wang L et al (2020) Mobile health technology to improve care for patients with atrial fibrillation. J Am Coll Cardiol 75:1523-1534. https://doi.org/10.1016/j.jacc.2020.01.052

34. Guo Y, Lane DA, Chen Y, Lip GYH (2020) Regular bleeding risk assessment associated with reduction in bleeding outcomes: the mAFA-II randomized trial. Am J Med 133:1195-1202.e2. https:// doi.org/10.1016/j.amjmed.2020.03.019

35. Chao T-F, Lip GYH, Lin Y-J et al (2018) Incident risk factors and major bleeding in patients with atrial fibrillation treated with oral anticoagulants: a comparison of baseline, follow-up and delta HAS-BLED scores with an approach focused on modifiable bleeding risk factors. Thromb Haemost 118:768-777. https://doi.org/ 10.1055/s-0038-1636534

36. Yoon M, Yang P-S, Jang E et al (2018) Dynamic changes of CHA2DS2-VASc score and the risk of ischaemic stroke in asian patients with atrial fibrillation: a nationwide cohort study. Thromb Haemost 118:1296-1304. https://doi.org/10.1055/s-0038-16514 82

37. Fauchier L, Bodin A, Bisson A et al (2020) Incident comorbidities, aging and the risk of stroke in 608,108 patients with atrial fibrillation: a nationwide analysis. J Clin Med 9:1234. https://doi. org/10.3390/jcm 9041234

38. Chao T-F, Lip GYH, Liu C-J et al (2018) Relationship of aging and incident comorbidities to stroke risk in patients with atrial fibrillation. J Am Coll Cardiol 71:122-132. https://doi.org/10. 1016/j.jacc.2017.10.085 